\title{
CARGA AÉREA EN EL FUTURO; EL AIRLANDER 10 Y EL AEROSCRAFT
}

Laura Alejandra Arias

Escuela de Negocios, Gestión y Sostenibilidad

Programa Tecnología en Gestión de Servicios para Aerolíneas

Giselle Camila Corredor Guzmán

Escuela de Negocios, Gestión y Sostenibilidad

Programa Tecnología en Gestión de Servicios para Aerolíneas

Daniela Estrada Herrera

Escuela de Negocios, Gestión y Sostenibilidad

Programa Tecnología en Gestión de Servicios para Aerolíneas

Paula Alejandra García Herrada

Escuela de Negocios, Gestión y Sostenibilidad

Programa Tecnología en Gestión de Servicios para Aerolíneas

María Fernanda Oliveros Contreras

Escuela de Negocios, Gestión y Sostenibilidad

Programa Tecnología en Gestión de Servicios para Aerolíneas

Felipe Sthewar Oyola Serrato

Escuela de Negocios, Gestión y Sostenibilidad

Programa Tecnología en Gestión de Servicios para Aerolíneas

Julián Camilo Pinto Rodríguez

Escuela de Negocios, Gestión y Sostenibilidad

Programa Tecnología en Gestión de Servicios para Aerolíneas

Kevin Sebastián Ruge Molina

Escuela de Negocios, Gestión y Sostenibilidad

Programa Tecnología en Gestión de Servicios para Aerolíneas

\section{Resumen}

En la actualidad, gracias a la globalización, el sector de carga aérea comercial ha tenido un gran impacto económico y competitivo a nivel global, donde se han implementado varios modelos de aviones, que pueden llegar a soportar grandes volúmenes de carga para facilitar el transporte de la misma con costos de movilización más rentables. Lo anterior, es de gran importancia y relevancia ya que las compañías constructoras de aeronaves dentro de los objetivos principales que tienen, es construir aeronaves que faciliten una innovación continua a precios bajos. En 
particular se hará comparación entre los fabricantes Hybrid Air vehicles que elabora el Airlander 10 y Aeros que produce el avión de carga Aeroscraft. Estas dos compañías se dedican a la innovación de la tecnología, buscando la mejora continua de aviones cargueros; El Airlander 10 tiene el propósito de entrar a competir con Aeroscraft en la implementación de nuevos modelos de aviones buscando ser competitivos con tarifas a nivel mundial; pensando siempre en el medio ambiente. Durante el desarrollo de este artículo se tratará como primera fase el origen, desarrollo y la producción de aeronaves que cumplan con las expectativas del mercado actual, con una visión a corto y mediano plazo, como segunda fase se hará un comparativo de las aeronaves que desarrollen las compañías Hybrid Air Vehicles y Aeros donde se evidencia cuál de los dos prototipos de aeronaves es más rentable y asequible a que sean comercializados en el mercado de las aerolíneas de carga a nivel mundial.

Palabras clave: Carga aérea, Prototipo, Aeronave, Fabricante. 


\section{Introducción}

Desde sus inicios la aviación ha ido presentando cambios en sus diseños de aviones para el transporte de pasajeros y mercancías, con el objetivo de mejorar cada vez más el servicio y la eficiencia que se presta por parte de estas compañías aéreas de carga. Hoy en día el sector carguero aéreo se encuentra en un ámbito muy competitivo, percibiendo el incremento industrial y teniendo en cuenta el gran impacto que han llegado a obtener a nivel global. El sector carguero está apostando a la propuesta de aviones híbridos no tripulados ya que estos tienen mayor capacidad para carga, y mayor autonomía de vuelo; son capaces de despegar y aterrizar de forma vertical en una superficie plana, reduce el impacto ambiental y su producción es de bajo costo.

Las compañías Hybrid Air vehicles y Aeros están en pro de mejorar el transporte aéreo de carga, estas compañías han diseñado, cada una un prototipo de avión el cual agiliza este servicio, tal es el caso del modelo "Airlander 10”, que está basado en el avance ecológico, tecnológico e innovador y el modelo "Aeroscraft" el cual consiste en un diseño innovador tanto en capacidad, tecnología y bajo consumo de combustible representando para el sector aeronáutico una gran importancia.

Debido a esto se ejecutarán 2 fases teniendo como base las compañías fabricantes Aeros y Hybrid Air vehicles; como variables de estudio identificando: El impacto ecológico, tecnológico y la economía que esta genera para el mercado de las aerolíneas cargueras y las ventajas y desventajas que tiene cada uno de los dos prototipos de aeronaves en cuanto a reducción de costos, combustible, autonomía de vuelo, eficacia, capacidad de carga y tecnología general. Finalmente, en la segunda fase a partir de los resultados encontrados, se realizará una comparación entre los dos prototipos. A partir de esta comparación se evidenciará cual es la aeronave que cumple con los puntos a evaluar y se seleccionará el diseño que cumpla con las mejores expectativas.

\section{Revisión de literatura}

La carga aérea hoy en día es importante para la economía y el comercio exterior de cada país, así mismo cada año va creciendo más. Según datos estadísticos del Banco Mundial, el transporte aéreo de carga demuestra un incremento del 2005 al 2017 aproximadamente de 95.333.04 toneladas (ORGANIZACION DE AVIACION CIVIL INTERNACIONAL , 2018), para llevar a cabo este movimiento de toneladas el sector aeronáutico debe contar con equipos adecuados para la realización de este procedimiento. Actualmente esta función la cumplen aeronaves tales como como el ANTONOV-AN 225 que tiene una capacidad de transportar 250 toneladas (AIR CHARTER SERVICE, 2018), el Beluga, del fabricante Airbus que tiene la aptitud de mover 155-144 toneladas (AIRBUS, 2018) y el Boeing 747-800 F con capacidad de 137 toneladas (BOEING, 2018).Estos aviones son unos de los encargados de transportar la carga aérea mundial. 
En el futuro se estima que se necesitará de un transporte masivo para estas mercancías que son transportadas constantemente y que son necesarias en distintos países; por lo que es necesario la creación de nuevas aeronaves con mayor capacidad de carga aérea, que brinden el transporte seguro de la carga, rapidez y eficacia en toda la operación de la misma, A continuación se mencionan los fabricantes que están diseñando prototipos para el funcionamiento adecuado de la carga en el futuro.

FABRICANTE AEROS: Nace en 1987 Ucrania, por Igor Pasternak es uno de los desarrolladores y fabricantes líderes de aeronaves de carga, certificado por FAA Aeronaves utilitarias y aerostatos (GREAT BIG STORY, 2016). Junto a su fundador y jefe de ingeniería, inician con la idea de crear un nuevo aerostato, con la capacidad de llevar carga y que tenga la característica de aterrizar en cualquier tipo de terreno para ayudas humanitarias en casos de emergencia especialmente en situaciones de desastres naturales. El 03 de enero de 2013 el Aeroscraft "Dragón Dream" se pone en pruebas de vuelo y de sistema en el hangar de la antigua estación aérea de la infantería marina en Tustin (California); la prueba resultó todo un éxito cumpliendo con los objetivos requeridos posteriormente su primer vuelo se realiza el 11 de septiembre del mismo año el cual tiene los resultados esperados para la compañía. El primer incidente que sufre la aeronave ocurre el 7 de octubre por cuestiones de infraestructura del hangar debido a que el techo del hangar en donde la aeronave se guardaba se derrumbó (POLSKY, 2016).

FABRICANTE HYBRID AIR VEHICLE: Es un fabricante de aeronaves híbridas fundado por Roger Munk en el año 2007; El Airlander 10, surge con idea innovadora y su objetivo principal es crear aeronaves más ligeras y ecológicas para tener la capacidad de volar durante semanas, enfocadas en el transporte de carga aérea (HYBRID AIR VEHICLES, 2018).

Inicialmente en Airlander 10, tiene la capacidad de carga de 10 toneladas, pero la compañía tiene la visión de poder producir una gama de aviones híbridos capaces de transportar hasta 1000 toneladas (HYBRID AIR VEHICLES, 2018). En 2016, el Airlander 10 da su primera demostración y posteriormente gana el título a la aeronave más grande del planeta. En cuanto a las pruebas de técnicas y de vuelo, la aeronave ha tenido complicaciones, teniendo 8 pruebas de las cuales 6 fueron exitosas y 2 fueron incidentes, lo cual preocupa a la compañía debido a que es un parámetro para lograr cumplir 200 horas de vuelo sin incidentes para que pueda obtener la licencia de operación comercial (ALVAREZ, 2018). Resaltando el impacto que estas aeronaves puede generar en el sector de la carga aérea, ya que dichas aeronaves poseen estructuras y sistemas más avanzados que las aeronaves de carga hoy en día, por lo tanto, estos diseños de aeronaves que aparte de contribuir con el medio ambiente, facilitan toda la operación desde la carga hasta la descarga.

\section{Metodología}

De acuerdo con la revisión de la literatura, se realiza una comparación entre las dos compañías fabricantes de estos prototipos de aviones de carga el Airlander 10 y el Aeroscraft, donde su objetivo principal es desarrollar aviones con una mayor capacidad de carga, buscando la reducción de costos de movilización de esta; como también la disminución de la emisión de 
gases contaminantes. La investigación realiza un análisis comparativo de las dos compañías que han implementado este diseño carguero a nivel mundial, como lo son Hybrid Air Vehicles y Aeros. A continuación, se explica cómo se han desarrollado estos prototipos:

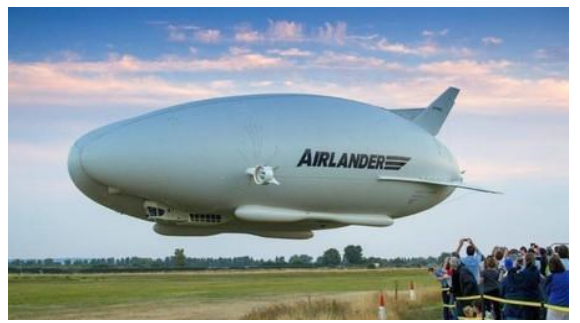

AIRLANDER 10 (Fig 1.): Para su sustentación, usa helio además de tener su estructura laminada y forma longitudinal curvada aerodinámica, en cuanto al sistema de aterrizaje, cuenta con neumáticos perfilados que proporcionan una operación en tierra en varias superficies incluyendo en agua. Posee 4 motores, dos en la parte delantera y dos en la popa, ya que esta configuración ayuda a la operación en crucero y la inyección es directa de diésel turboalimentado (HYBRID AIR VEHICLES, 2018).

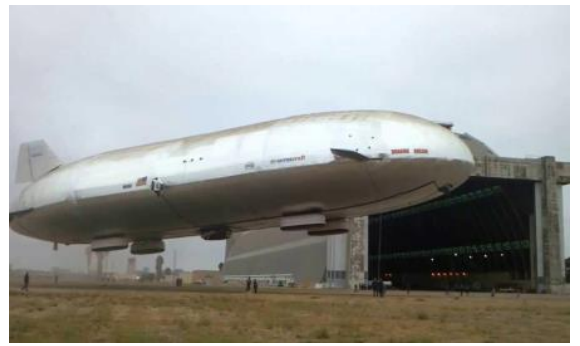

Fig. 2 Aeroscraft Fuente: Google (2019)
AEROSCRAFT (Fig 2.): Este vehículo posee un sistema llamado $\mathrm{COSH}$, que permite operar como un vehículo más liviano que el aire, así como también permite presurizar el helio (AEROS COMPANY, 2018), su estructura es rígida, hecha en fibra de carbono ultraligera (AEROS COMPANY, 2018). Tiene motores vectoriales que ayudan a maniobrar en forma helicóptero (AEROS COMPANY, 2018). En cuanto a su aterrizaje, este tiene un sistema parecido a los submarinos ya que este puede tomar o expulsar aire para pesar más o pesar menos, esto permite su aterrizaje vertical (AEROS COMPANY, 2018).

\section{Resultados preliminares}

A partir de la revisión documental realizada por el grupo y la comparación entre los dos fabricantes se hallaron los siguientes resultados:

\begin{tabular}{|c|c|c|}
\hline Características & AIRLANDER 10 & AEROSCRAFT ML866 \\
\hline Longitud & $92 \mathrm{~m}(302 \mathrm{pies})$ & $\begin{array}{c}555 \text { Pies } \\
(169.16 \mathrm{Metros})\end{array}$ \\
\hline Envergadura & $43.5 \mathrm{~m}(143 \mathrm{pies})$ & $\begin{array}{c}177 \text { Pies } \\
(53.9 \mathrm{Metros})\end{array}$ \\
\hline Altura & $26 \mathrm{~m}(85 \mathrm{pies})$ & $\begin{array}{c}120 \text { Pies } \\
(36.5 \mathrm{Metros})\end{array}$ \\
\hline Capacidad de carga & Hasta $10,000 \mathrm{~kg}$ & $\begin{array}{c}\text { Hasta } 66 \text { Toneladas } \\
(147.840 \mathrm{lbs})\end{array}$ \\
& $(22,050 \mathrm{lbs})$ & \multicolumn{2}{|c}{} \\
\hline
\end{tabular}




\begin{tabular}{|c|c|c|}
\hline Altitud de crucero & Hasta 20,000 pies $(6,096 \mathrm{~m})$ & $\begin{array}{c}\text { 12.000 Pies } \\
\text { (3657.6 Metros ) }\end{array}$ \\
\hline Velocidad Máxima & $\begin{array}{c}20 \text { nudos de holgazanería } \\
(37 \mathrm{~km} / \mathrm{hr})\end{array}$ & 120 Nudos ( 222.24 km/h) \\
\hline Velocidad crucero & 80 nudos $(148 \mathrm{Km} / \mathrm{h})$ & 100 Nudos ( $185.2 \mathrm{Km} / \mathrm{h})$ \\
\hline Tipo de estructura & $\begin{array}{c}\text { Casco relleno de helio, } \\
\text { construcción de tejido } \\
\text { laminado. }\end{array}$ & $\begin{array}{l}\text { Rígida hecha de aluminio } \\
\text { ultraligero y fibra de carbono }\end{array}$ \\
\hline Motores & $\begin{array}{l}4 \text { motores V8 diesel que } \\
\text { permiten empuje vectorial } \\
\text { para despegue, aterrizaje, } \\
\text { manejo en } \\
\text { operación. }\end{array}$ & $\begin{array}{l}\text { Motores de empuje } \\
\text { Vectoriales }\end{array}$ \\
\hline $\begin{array}{l}\text { Rango máximo con } \\
\text { carga útil }\end{array}$ & $20,000 \mathrm{~kg}(44,100 \mathrm{lbs})$ & $\begin{array}{l}\text { 3,100 Millas náuticas } \\
\text { (5741.2 Kilometros ) }\end{array}$ \\
\hline Tipo de despegue & Horizontal & Vertical \\
\hline Tren de aterrizaje & Patines de aterrizaje & Cojines de aterrizaje \\
\hline
\end{tabular}

Tabla 1 Comparación entre los fabricantes

Fuente: Propia (2019)

A Partir de la relación entre la comparación del Airlander 10 y aeroscraft se concluye que el prototipo idóneo para la carga en el futuro es el aeroscraft, ya que este prototipo abarca las variables de estudio planteadas para seleccionar el mejor prototipo. estas variables son, la tecnología, la economía y la ecología que este prototipo le aporta a las aerolíneas cargueras ya que su tecnología y características físicas, hacen que sea la opción más viable para ser implementado en el sector de la carga aérea, por ende en su fabricación los factores determinantes que llevaron a elegir esta opción, se establecen como: su tamaño que es de 555 $\mathrm{ft}$ que hace que este híbrido se movilice a $220 \mathrm{~km} / \mathrm{h}$ y con solo usar 7200 Galones de combustible (27254.96 Litros) que equivale a 1/5 en cuanto a diferencia del consumo de los aviones cargueros que se encuentran actualmente en el sector, utiliza también el helio ayudando 
a su sostenibilidad para que se prolongue por más tiempo; en cuanto su capacidad de carga corresponde de 66 a 500 toneladas dependiendo el prototipo, este no tiene necesidad de contar con una superficie plana para despegar y aterrizar, ya que el tren de aterrizaje no es igual al que se utiliza hoy en día, este implementa almohadillas que incluso pueden ayudar a aterrizar en el agua cuenta con 3 turbinas vectoriales que ayudan al empuje y seis turbohélices en su parte inferior que controlan el equilibrio y movimiento del modelo. 


\section{Conclusiones}

El transporte de carga aérea en el futuro y sus aeronaves, está puesta en la facilitación de procesos, competitividad, responsabilidad ecológica y avances tecnológicos para así ser más rentables a nivel mundial, históricamente y hasta la actualidad, el transporte de carga aérea ha tenido gran impacto a nivel mundial, por lo tanto, es de esperarse que el sector carguero se esmere en tener sistemas y procesos más avanzados en tecnología, el fortalecimiento de los procesos cargueros demuestra que las empresas del sector aéreo están comprometidas con la tecnología, desarrollo y sostenibilidad en avances para mejorar el transporte de carga aérea en el futuro.

Airlander 10 y Aeroscraft, son excelentes opciones para el futuro de la carga aérea, porque su objetivo principal está centrado en la innovación aérea sumándole valores agregados al mercado. El transporte de la carga por vía aérea está comprometido con la industria, dando a conocer procesos de fabricación de sus aviones con mayor capacidad de carga. Esto es un futuro, dará mayor calidad y agilidad en el servicio. La distribución de la carga dentro de la aeronave es de vital importancia, por ello contar con aeronaves que puedan transportar más toneladas, bajando considerablemente el consumo de combustible y a mayor velocidad ayudando a la sostenibilidad implementa un avance favorecedor en la industria. 


\section{Referencias}

AEROS COMPANY . (2018). Aplicaciones comerciales . Obtenido de http://aeroscraft.com/applications/4580412718

AEROS COMPANY . (2018). Capacidades. Obtenido de http://aeroscraft.com/capabilitiescopy/4580476906

AEROS COMPANY . (2018). FLOTA. Obtenido de http://aeroscraft.com/fleet-copy/4580475518

AEROS COMPANY. (2018). CONTROL DE LA SANIDAD ESTÁTICA (COSH). Obtenido de http://aeroscraft.com/technology-copy/4580412172

AEROS COMPANY. (2018). ESTRUCTURA RÍGIDA. Obtenido de http://aeroscraft.com/technologycopy/4580412172

AEROS COMPANY. (2018). MOTORES DE VELOCIDAD VECTORIALES. Obtenido de http://aeroscraft.com/technology-copy/4580412172

AEROS COMPANY. (2018). PRINCIPALES COMPONENTES DE COSH. Obtenido de http://aeroscraft.com/technology-copy/4580412172

AEROS COMPANY. (2018). Tecnologia clave. Obtenido de http://aeroscraft.com/technologycopy/4580412172

AIR CHARTER SERVICE. (2018). ANTONOV AN-225. Obtenido de https://www.aircharterservice.com/aircraft-guide/cargo/antonov-ukraine/antonovan-225

AIRBUS. (2018). Beluga. Obtenido de https://www.airbus.com/aircraft/freighter/beluga.html\#airlifter

ALVAREZ, R. (5 de 08 de 2018). Así de lujoso es el interior del Airlander 10, la aeronave más grande del mundo. Obtenido de XATAKA: https://www.xataka.com/vehiculos/asi-lujosointerior-airlander-10-aeronave-grande-mundo

BOEING. (2018). Especificaiones tecnicas. Obtenido de https://www.boeing.com/commercial/freighters/

GREAT BIG STORY (Dirección). (2016). Blimp My Ride: Making It Big with a Blimp Tycoon [Película].

HYBRID AIR VEHICLES. (2018). Introduccion . Obtenido de Detalles tecnicos :

https://www.hybridairvehicles.com/aircraft/airlander-10

HYBRID AIR VEHICLES. (2018). INTRODUCCION . Obtenido de https://www.hybridairvehicles.com/aircraft/airlander-10

HYBRID AIR VEHICLES. (2018). Nuestra historia . Obtenido de https://www.hybridairvehicles.com/about-us/our-story 
HYBRID AIR VEHICLES. (2018). Nuestra Vision. Obtenido de https://www.hybridairvehicles.com/about-us/our-vision

NEXT BIG FUTURE. (2018). Aeronaves híbridas acercándose a una importante comercialización. Obtenido de https://www.nextbigfuture.com/2016/03/hybrid-airships-nearing-significant.html

ORGANIZACION DE AVIACION CIVIL INTERNACIONAL . (2018). Transporte aéreo, carga (millones de toneladas-kilómetros). Obtenido de Transporte aéreo, carga (millones de toneladas-kilómetros)

POLSKY, G. (Dirección). (2016). Inventing a Lighter-Than-Air Aircraft [Película]. 